führten Wirtschaftssubjekten den normativen Rahmen ihres Marktverhaltens setzen. Das seit 1979 schnell wachsende wirtschaftsbezogene Recht der VR China dokumentiert diesen Vorgang. Die Bücher von Wolff und Zheng wenden sich an Interessierte, die einen einführenden Überblick suchen. Beide bleiben unvermeidlich kurz zu den behandelten Sachgebieten; die Fülle des einbezogenen Stoffes läßt oft nur zu, einschlägige Normen zu erwähnen, ohne sie näher zu erörtern. Wolff schreibt dabei im Stil eines Leitfadens mit Blick auf den in China tätigen ausländischen Kauf mann, Zheng legt den Schwerpunkt auf rechtswissenschaftlichen systematischen Gesamtaufbau seiner Darstellung. Hinweise auf Spezialliteratur erlauben, Einzelfragen näher nachzugehen. In Zhengs - recht knapper - Literaturliste (S. $361 \mathrm{f}$.) sind Verfasser alphabetisch, jedoch bunt durcheinander, mal nach Vor-, mal nach Familiennamen geordnet. Außerdem wird für chinesisch-sprachige Literatur nicht der jeweilige Titel in Latein-Lautschrift (etwa der in der VR China üblichen pinyin-Umschrift) mitgeliefert, was erschwert, die originalen Werke zu identifizieren. Dies schafft besonders bei anscheinend unpräzisen Eindeutschungen Verwirrung (vgl. a.a.O., "Amtsblatt des Staates der VR China [sic]; ist dies der Guowuyuan gongbao, mithin das "Amtsblatt des Staatsrats der VR China"?). Tatsächliche Umstände einzelner Rechtsgebiete werden sehr ungleichgewichtig, mitunter gar nicht behandelt. Wer z.B. die wenigen Zeilen bei Zheng zu "Schmuggel" (S. 304) liest, erfährt nichts über das kolossale Ausmaß des Phänomens in der VR China oder über die Verquickung der Streitkräfte in diesen Schwarzhandel. Beide Schriften sind gewiß begrüßenswerte Neuerscheinungen, die dem uneingeweihten Leser den zunehmend wichtigen Gegenstand des chinesischen Wirtschaftsrechts näherbringen. Tiefe im Detail wird der an konkreten Rechtsfragen Interessierte aber woanders suchen müssen, auch weil - worauf in beiden Arbeiten einleitend zu Recht hingewiesen wird besonders dieser Bereich des chinesischen Rechts sich nach wie vor rasch verändert.

\title{
Wolfgang Kessler
}

\section{Emily Hahn-Godeffroy}

Die südaf rikanische Truth and Reconciliation Commission

Nomos Verlagsgesellschaft, Baden-Baden, 1998, 293 S., DM 88,--

Am 28. Juni 1995 verabschiedete das südafrikanische Parlament nach über 130 Stunden Beratungen im Rechtsausschuß und mehr als 300 Entwurfsänderungen das Gesetz zur Förderung der nationalen Einheit und Versöhnung (Promotion of National Unity and Reconciliation Bill). Ziel des Gesetzes ist, durch Untersuchungen und umfassende Aufdeckung (full disclosure) von schweren Menschenrechtsverletzungen die Einheit und Versöhnung des südafrikanischen Volkes zu fördern. Es basiert auf dem Grundsatz, daß Versöhnung von Vergebung abhängt, Vergebung aber erst dann stattfinden kann, wenn die 
Wahrheit über schwere Menschenrechtsverletzungen bekannt wird (Explanatory Memorandum to the Parliamentary Bill. ${ }^{1}$

Zuständig für die Untersuchungen ist die Wahrheits- und Versöhnungskommission (Truth and Reconciliation Commission - TRC). Emily Hahn-Godeffroy stellt im Hauptteil ihrer Dissertation die grundlegende Konzeption und Aufgabenbereiche dieser Kommission dar.

Die Aufgaben der TRC umfassen gemäß § 3 I des Gesetzes für Nationale Einheit und Versöhnung die Darstellung der zwischen dem 01.03.1960 (Jahr des gesetzlichen Verbots der Freiheitsbewegungen und Aufnahme des bewaffneten Kampfes gegen das Apartheidregime, Monat des Sharpeville-Massakers) und dem 10.05.1994 (Tag der Amtseinsetzung von Präsident Mandela) begangenen schweren Menschenrechtsverletzungen sowie die Gewährung von Amnestien gegenüber denjenigen Personen, die innerhalb dieser Frist begangene politisch motivierte Taten vollständig offenlegen und die sonstigen Anforderungen des Gesetzes erfüllen. Ferner soll durch die Aufdeckung des Schicksals der Opfer von Menschenrechtsverletzungen ihre Würde wiederhergestellt und Empf ehlungen von Maßnahmen zur Wiedergutmachung und Rehabilitierung ausgearbeitet werden. Schließlich hat die TRC einen Abschlußbericht zu erstellen.

Aus diesen Aufgaben ergibt sich ihre Aufgliederung in drei Ausschüsse: 1. den Menschenrechtsausschuß, 2. den Amnestieausschuß und 3. den Wiedergutmachungs- und Rehabilitierungsausschuß. Diese Konzeption zeigt, "daß die Aufdeckung der Wahrheit und die Versöhnung des Volkes diejenigen Ziele waren, denen unter den derzeitigen politischen Umständen Südafrikas die herausragende Bedeutung zugemessen wurde" (S. 43). Zurücktreten mußte hingegen die Forderung nach "Gerechtigkeit" und insbesondere die strafrechtliche Verfolgung der Täter, da dem geständigen Täter Amnestie gewährt wird.

Die Amnestieregelungen sind denn auch höchst umstritten und waren bereits Gegenstand einer Klage vor dem südafrikanischen Verfassungsgericht, was die Autorin analysiert und bewertet. Die politische Organisation AZAPO sowie weitere Angehörige von Opfern legten am 9. April 1996 dem Verfassungsgericht einen Antrag auf Feststellung der Verfassungswidrigkeit des Gesetzes für Nationale Einheit und Versöhnung vor. Sie rügten insbesondere die Regelungen des $\S 20$ VII, der die strafrechtliche, wie auch die zivilrechtliche Haftung im Falle der Amnestiegewährung ausschließt, als einen Verstoß gegen die verfassungsmäßige Rechtsschutzgarantie (Art. 22 der Übergangsverfassung). In seinem Urteil vom 25. Juli 1996 stellte das Verfassungsgericht fest, daß ein Eingriff in Art. 22 ÜVerf. zwar vorliege, aber gemäß Art. 33 II durch die Schlußbestimmung der Übergangsverfassung, die den Grundgedanken der Nationalen Einheit und Wiedergutmachung und die Gewährung von Amnestien formuliert, gerechtfertigt sei (The Azanian People's Organization (AZAPO) v. President of the Republic of South Africa, CCT 17/96). Die Autorin sieht in dem Urteil trotz einer extensiven teleologischen Auslegung des Begriffs der Amnestie und einer "stark politisch orientierten Argumentation" (S. 132) einen wichtigen Beitrag des Verfassungsge- 
richts zur Erhöhung der Akzeptanz der zukünftig gewährten Amnestien und damit zugleich eine "grundlegende Bestätigung von Sinn und Zweck der TRC" (S. 133).

Abschließend nimmt Emily Hahn-Godeffroy eine Bewertung der TRC vor, wobei sie drei verschiedene Anknüpfungspunkte zugrunde legt: Zunächst untersucht sie, ob die TRC in funktionaler Hinsicht in der Lage ist, die Aufarbeitung von Vergangenheit zu leisten. Hieran schließt sich eine zusammenfassende Darstellung der deutschen Vergangenheitsbewältigung an, um schließlich mit Hilfe eines Vergleichs beider Wege die Besonderheiten der südafrikanischen Wahrheitskommission herauszuarbeiten.

Die herausragenden Komponenten der TRC sieht sie zum einen in der Aufdeckung der Wahrheit, wozu nicht nur die realitätsgetreue, umfassende Darstellung der Schicksale der Opfer zählt, sondern auch eine genaue Analyse der Strukturen, Hierarchien und Befehlsketten des Apartheidsystems. Die zweite bedeutende Komponente stelle die Amnestiegewährung dar. Da nur der voll geständige Täter auf eine Amnestie hoffen kann, wird der "Täter selbst in den Dienst der Wahrheitsfindung" (S. 180) gestellt. Allerdings, so merkt die Autorin kritisch an, funktioniert dieses Prinzip "Amnestie gegen Geständnis" zum Zeitpunkt ihrer Untersuchungen im Januar 1997 noch nicht in dem gewünschten Maße. Viele Täter würden die Entwicklungen abwarten, diejenigen, die bisher einen Antrag auf Amnestiegewährung gestellt haben, seien Häftlinge oder hätten in nächster Zeit die Erhebung der öffentlichen Anklage durch die Staatsanwaltschaft zu befürchten. Die Autorin sieht daher die Wahrheitskommission "bisher in erster Linie als eine von Opfern dominierte Institution (...), welcher sich die Täter entziehen" (S. 181).

In Deutschland wurde die Aufarbeitung der Vergangenheit zum einen durch die Einsetzung einer Enquête-Kommission des Bundestages sowie des Bundesbeauftragten für die Unterlagen des Staatssicherheitsdienstes der ehemaligen DDR (sog. Gauck-Behörde) gem. StasiUnterlagen-Gesetz von 1994 geleistet. Beide Institutionen haben sich nach Ansicht der Autorin hinsichtlich der "Aufdeckung der Wahrheit" als förderlich erwiesen und einen "wesentlichen, unverzichtbaren Beitrag zur Vergangenheitsaufarbeitung des SED-Unrechts" (S. 198) geleistet. Die strafrechtliche Verfolgung der Täter von Menschenrechtsverletzungen ist "nicht nur theoretische Forderung geblieben (...), sondern (hat) in der Praxis bereits zu einigen Verurteilungen nicht nur der einfachen Grenzsoldaten, sondern auch führender verantwortlicher Politiker in der DDR (geführt)." (S. 204).

Eine Bewertung der TRC aus deutscher Sicht ergibt nach Ansicht der Autorin, daß sich "Südafrika für einen sehr unkonventionellen Weg der Vergangenheitsaufarbeitung entschieden" habe (S. 204). Ein wesentlicher Unterschied beider Aufarbeitungssysteme bestehe bereits in der Konzeption der südafrikanischen TRC selbst, die als alleinige Institution mit der Vergangenheitsaufarbeitung betraut sei, während in Deutschland eine scharfe Trennung zwischen der Aufdeckung der Wahrheit durch die Enquête-Kommission und die Gauck-Behörde einerseits, und der Sanktionierung des Unrechts durch nationale Gerichte andererseits bestehe. Die zentrale Auseinandersetzung mit der Vergangenheit in Südafrika habe aber den Vorteil, daß der Komplexität der Thematik Rechnung getragen werden kann und die einzelnen Elemente wie Wahrheitsfindung und Reparationsmaßnahmen besser 
koordiniert werden könnten. Auch zeige sich, daß in Südafrika in weit höherem Maße die Bevölkerung in den Aufarbeitungsprozeß integriert sei, während in Deutschland die staatliche Initiative dominiere. Dies hänge damit zusammen, daß dieser Prozeß in Deutschland eher als ein juristischer, denn als gesellschaftspolitischer Prozeß gesehen werde. Auffällig sei darüber hinaus die vorrangige Stellung der Wahrheitsfindung in Südafrika, für die letztendlich die Sanktionierung des begangenen Unrechts "geopfert" würde (S. 206). In Deutschland hingegen bestehe keine derartige Gewichtung, sondern beide Elemente bildeten zwei unterschiedliche und unabhängige Elemente der Aufarbeitung.

In ihrem kurzen Ausblick auf die Eignung der TRC zur Förderung der Versöhnung kommt die Autorin zu dem Ergebnis, daß "die TRC durch ihren wertvollen Beitrag zur Aufdeckung der Wahrheit in jedem Fall eine überragend wichtige Grundvoraussetzung für die Versöhnung des südafrikanischen Volkes schafft" (S. 211). Die Versöhnung könne nicht allein durch die Arbeit der TRC herbeigeführt werden, sondern sei ein langwieriger gesellschaftlicher Prozeß, der selber "wachsen" müsse.

Das vorliegende Werk bietet eine wertvolle Zusammenfassung der Konzeption, Aufgabengebiete und Arbeitsweisen der TRC. Verbunden mit den Hintergrundinformationen zur Apartheidpolitik und den Wahrheitskommissionen in Lateinamerika, die wesentlichen Einfluß auf die südafrikanische Wahrheitskommission hatten, stellt sich die Dissertation als wichtige Informationsquelle bei der Bewertung des inzwischen am 29. Oktober 1998 erschienenen Abschlußberichts der südaf rikanischen Wahrheits- und Versöhnungskommission dar.

Peggy Wittke

\section{Stefanie Pautke}

Die kartellrechtliche Erfassung konglomerater Konzentration in der Republik

\section{Südaf rika}

Schriftenreihe Recht und Verfassung in Südafrika, Band 2 (Herausgegeben von Ulrich

Karpen, Ingo von Münch und Hans-Peter Schneider)

Nomos Verlagsgesellschaft, Baden-Baden, 1998, 208 S., DM 66,--

Nicht nur die demokratischen Strukturen am Kap der Guten Hoffnung unterliegen einem mühsamen (noch nicht abgeschlossenen) Entwicklungsprozeß; auch die Wirtschaftsverfassung Südafrikas muß langsam ihren Sonderweg verlassen und zu vergleichbaren Formen moderner Volkswirtschaften aufschließen. Die speziellen Umstände der Besiedlung, der politischen Herrschaft und der Industrialisierung haben dort ebenso tiefe Spuren gegraben wie die Zeit der Apartheid, in der sich westliche Investoren aus ihren Engagements zurückzogen. Das Diktat des Überlebens diktierte damals auch die Entstehung oligopoler Machtgebilde in der Wirtschaft. 